\title{
Interferometric time division FBG interrogator and multiplexer with static, dynamic, and absolute wavelength measurement capabilities
}

\author{
Philip Orr*, Alan Paterson and Paweł Niewczas \\ Institute for Energy and Environment, University of Strathclyde, Glasgow G1 1XW, UK
}

\begin{abstract}
We report on the design and preliminary testing of an interferometric interrogator capable of large-scale time-division multiplexing of FBG sensors. The scheme employs a passive algorithm for phase demodulation, allowing changes in FBG sensor reflected wavelengths to be calculated instantaneously upon arrival, and incorporates a technique for identification of initial absolute sensor wavelengths in order to overcome the measurement ambiguity associated with interferometric schemes. The proposed system will allow for high-speed interrogation of large-scale FBG sensor arrays with interferometric resolution and the capability for dynamic, static, and absolute FBG wavelength measurement.
\end{abstract}

Keywords: Fibre Bragg grating, interrogation, interferometry, time division multiplexing, absolute wavelength

\section{INTRODUCTION}

Interferometric interrogation, while offering the highest resolution of all techniques ${ }^{1,2}$, can be difficult to integrate with existing fibre Bragg grating (FBG) multiplexing schemes due to the requirement for either stabilisation or modulation of the optical path difference (OPD). As such, it is most commonly utilised in single-sensor schemes for high-frequency, low-amplitude measurements. Conventional interrogation systems therefore possess a trade-off between their extensibility (number of sensors the scheme can interrogate) and the quality of the measurement (accuracy, resolution, and rate of measurement) ${ }^{3-5}$. Hybrid interferometric wavelength-division multiplexing (WDM) schemes have, however, been successfully developed in attempts to address this trade-off. Of particular note are the schemes by Berkoff ${ }^{6}$ and Johnson $^{7}$; the former combining passive WDM with active OPD modulation, and the latter utilising an instantaneous phase demodulation technique ${ }^{8}$ in order to deploy a continuously-scanning spectral filter. An interferometric timedivision multiplexing (TDM) system has also been demonstrated by Weis ${ }^{9}$. Although succeeding in combining interferometric FBG shift tracking with WDM/TDM techniques, these approaches retain performance limitations stemming from the need for active OPD modulation or the use of tunable components, resulting in limitations in, respectively, quasi-static measurement capability or extensibility of the sensor array.

In this paper we build upon the schemes discussed above in order to demonstrate a passive interferometric time-division multiplexing FBG interrogator for the high-resolution interrogation of large-scale sensor arrays. In order to overcome the ambiguity in absolute wavelength associated with interferometric schemes, we describe a technique for calibration of absolute initial sensor wavelengths that may be deployed in any multiplexing passive-interferometric interrogator. The preliminary laboratory demonstration of both techniques is reported.

\section{INTERROGATION SYSTEM DESIGN}

A diagram of the proposed system is shown in Figure 1. Each grating reflects within the bandwidth of the CW broadband source, which is pulsed periodically. Grating wavelengths need not be unique, and are discriminated between by their time of arrival at the interrogator which is set by the delay time $\tau$. The passive phase-demodulation algorithm utilising a $3 \times 3$ coupler based Mach-Zehnder interferometer (MZI) developed by Todd et al ${ }^{8}$ is deployed in order to instantaneously determine the change in reflected wavelengths upon arrival using a single sample on each photodetector. Sampling and digitization of held peak photodetector outputs is triggered by an intensity threshold. The ADC device also gates a pulse generator, driving the modulated source at the desired interrogation rate. In combination with the calibration procedure in Section 3, the system is thus able to track absolute dynamic or quasi-static measurands.

*philip.orr@strath.ac.uk; phone +44 (0)141 548 4841; fax +44 (0)141 548 4872; www.instee.strath.ac.uk 


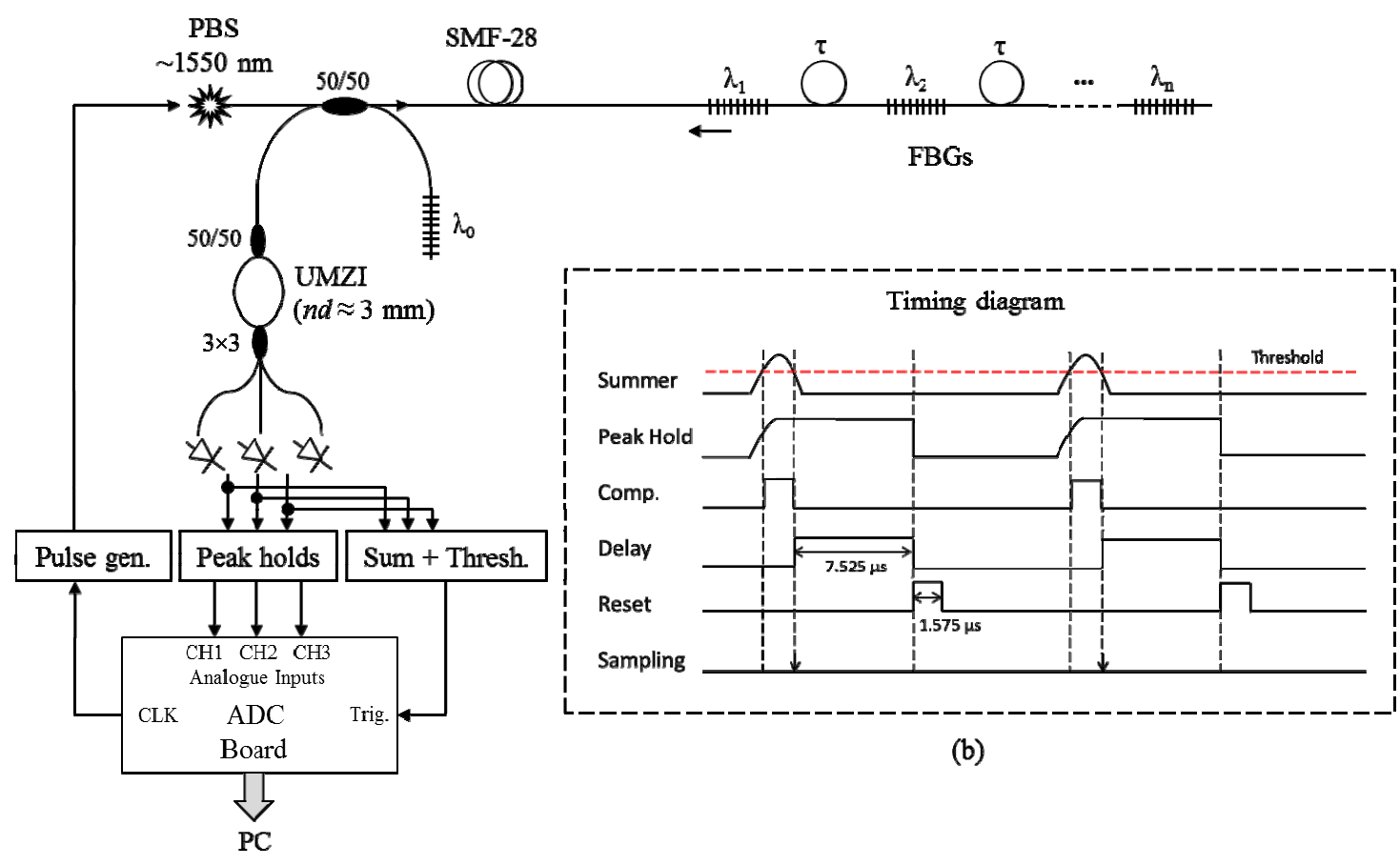

(a)

Figure 1. (a) Interferometric time-division multiplexing FBG interrogation scheme: A pulsed broadband source (PBS) illuminates all FBGs, with reflection arrivals staggered by delays of $2 \tau$. UMZI is unbalanced Mach-Zehnder interferometer. (b) Timing diagram for electronic data capture scheme showing a single peak hold which is reset following digitization.

In our preliminary laboratory scheme, the pulsed source is configured with a repetition rate of $2 \mathrm{kHz}$. Peak hold resetting is performed approximately $9 \mu$ s following the arrival of grating reflections, as illustrated in Figure 1(b). All grating wavelengths are situated within the telecoms C-band $(\sim 1550 \mathrm{~nm})$. The unbalanced MZI is an all-fibre construction, fabricated between the output pigtails of $1 \times 2$ and $3 \times 3$ couplers by fusion splicing. To isolate the MZI from vibrations, the device was bonded to a steel plate. A National Instruments PXI unit was used as the central controller, facilitating the simultaneous sampling of three analogue channels as triggered by the threshold input, and driving the pulse generator by TTL clock.
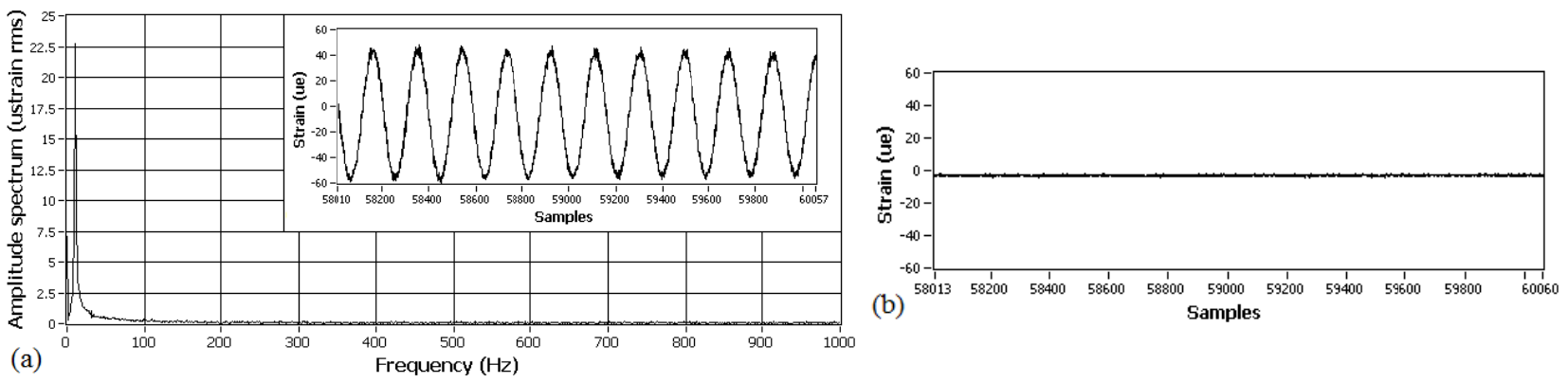

Figure 2. (a) Demonstration of spectral noise floor $(\sim 100 \mathrm{n} \varepsilon / \sqrt{\mathrm{Hz}}$ in present laboratory embodiment) and sinusoidal signal applied at $10 \mathrm{~Hz}$. The lack of cross-talk to a second FBG sensor is evident from (b), recorded simultaneously.

Figure 2 (a) and (b) illustrate the performance of the present embodiment. The spectral noise density of the technique, measured during a quiescent period, is presently approximately $100 \mathrm{n} \varepsilon / \sqrt{ } \mathrm{Hz}$. This is an order of magnitude worse than the achievable performance in interferometric multiplexers that do not use intermediate electronic subsystems ${ }^{10}$, and can be improved in future through circuit re-design. However, this noise level still represents an order of magnitude improvement over commercial strain gauge schemes. From Figure 2(b), recorded during application of a $\sim 30 \mu \varepsilon \mathrm{rms}$ 
signal to a single sensor (Figure 1(a)), it is clear that there is no cross-talk between sensors multiplexed using this approach. The key features of the proposed scheme are its large-scale extensibility, its retention of interferometric accuracy and resolution, and its capability for absolute measurement (discussed in the next Section) - a combination that differentiates it from existing interrogation systems. Although demonstrated here with a small number of sensors, the scheme may be expanded to handle large sensor arrays, and the interrogation rate (here limited by the response/reset time of the bespoke electronic subsystems) may be greatly increased in future iterations.

\section{INITIAL WAVELENGTH CALIBRATION}

Although interferometric interrogation schemes for FBG sensors can offer the highest dynamic and quasi-static resolutions ${ }^{11}$, it is often the case that measurements are referred to the state of the sensors at "switch-on time" due to the cyclic nature of phase demodulation. In many applications it is desirable to be able to determine remotely and immediately the absolute value of the sensor reflected wavelengths, to determine for example the temperature or slowlychanging strain in a structure, which may have fluctuated since the last observation. Here we describe a technique for determining initial FBG wavelengths in a remote sensor array during the start-up procedure of any multiplexing passiveinterferometric interrogator, and report on a preliminary laboratory implementation of the scheme as used to augment the TDM interrogator described above.

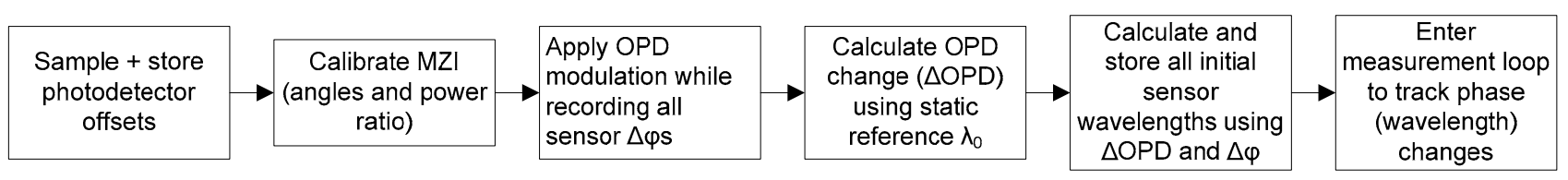

Figure 3. Start-up operational procedure for calibration of photodetector offsets, MZI parameters, and initial sensor wavelengths using a multiplexing interferometric interrogator.

The procedure, illustrated in Figure 3, follows after photodetector and MZI calibration ${ }^{12}$, and relies on brief modulation of the optical path difference in the MZI - which is thereafter held steady during the primary measurement stage. Using a local static wavelength reference in the form of an athermal FBG, or an FBG configured to track an absorption line ${ }^{13}$, the chosen multiplexing technique may be used to monitor the phase change $\Delta \varphi$ on all FBG channels. Due to the difference in wavelengths, the relationship $\delta \varphi / \delta \mathrm{OPD}$ will be unique for each grating. For the case of the static reference, its wavelength $\lambda_{0}$ is known and thus the MZI modulation $\delta$ OPD may be calculated using

$$
\delta \mathrm{OPD}=\lambda_{0} \delta \varphi_{0} / 2 \pi
$$

where $\delta \varphi_{0}$ is the phase change recorded for the static grating at $\lambda_{0}$. Following this, the $\Delta \varphi$ for all sensor gratings may be used with the common $\delta$ OPD to calculate all initial wavelengths $\lambda_{1-n}$ using

$$
\lambda_{1-\mathrm{n}}=2 \pi \delta \mathrm{OPD} / \delta \varphi_{1-\mathrm{n}}
$$

After initial wavelengths have been determined, the static reference may be tracked in order to identify drifts in the OPD such that phase-wavelength conversion is accurate. In order to demonstrate the technique, an unbalanced (nominal OPD of $3 \mathrm{~mm}$ ) MZI was constructed on a metal plate with no acoustic or thermal isolation. A combination of heating and bending of the paths was then utilized to shift the OPD by up to $50 \mu \mathrm{m}$. A stabilized FBG was used to provide the wavelength reference $\lambda_{0}$, situated at $1539.56 \mathrm{~nm}$, while a "sensing" FBG $\lambda_{1}$ was held steady at $1554.66 \mathrm{~nm}$. Following the procedure in Figure 3, modulation of the OPD was applied during the simultaneous monitoring of both FBGs, facilitated by the TDM scheme described in Section 2. Calculation of $\lambda_{1}$ was then performed using $\delta \varphi_{0}$ and $\delta \varphi_{1}$. In this preliminary test, $\lambda_{1}$ could be determined to within the accuracy of $2 \mathrm{~nm}$. Accuracy is determined by a combination of factors: Variability and hence inaccuracy in the "static" wavelength over the modulation period; noise level on both phase measurements; and slow OPD modulation. The development of a stable, fast, repeatable OPD modulation technique, proper stabilization of the wavelength reference, and improved electronic noise contributions, will allow wavelength accuracy to be greatly improved in future iterations, particularly since this technique assumes that the OPD modulation has a temporal characteristic much faster than sensor perturbations over this period, such that sensor wavelengths can be assumed to be static over the duration of the OPD modulation. 


\section{CONCLUSIONS}

In this paper we have introduced both a TDM interferometric FBG interrogation scheme and an augmenting technique for determination of absolute sensor wavelengths. In this way, the extensibility of TDM topologies is combined with the accuracy and resolution of interferometric wavelength shift detection and the capability for dynamic, quasi-static, and absolute measurement capability. A preliminary laboratory demonstration of the combined system was reported, operating at $2 \mathrm{kHz}$ and yielding a spectral noise density of $100 \mathrm{n} \varepsilon / \sqrt{ } \mathrm{Hz}$ on each sensor and no detectable cross-talk. The absolute wavelength calibration technique yielded an accuracy of $2 \mathrm{~nm}$, limited primarily by stabilization issues which will be improved in future iterations. Notably, the absolute initial wavelength technique is relevant to all multiplexing passive-interferometric interrogators, allowing any such scheme to intrinsically and straightforwardly determine initial sensor wavelengths without relying on additional components.

\section{REFERENCES}

[1] D. Kersey et al, "Fiber Grating Sensors," J. Lightwave Tech. 15(8):1442-1463 (1997)

[2] A. D. Kersey, T. A. Berkoff, and W. M. Morey, "High resolution fiber Bragg grating based strain sensor with interferometric wavelength detection," Electronic Letters 28(236):237 (1992)

[3] A. Ezbiri, S. E. Kanellopoulos, and V. A. Handerek, "High resolution instrumentation system for fibre-Bragg grating aerospace sensors," Opt. Comm. 150:43-48 (1998)

[4] C. Boulet, D. J. Webb, M. Douay, and P. Niay, "Simultaneous interrogation of fiber Bragg grating sensors using an acoustooptic tunable filter," Phot. Tech. Lett. 13(10):1215-1217 (2001)

[5] A. D. Kersey, T. A. Berkoff, and W. M. Morey, "Multiplexed fiber Bragg grating strain-sensor system with a fiber Fabry-Perot wavelength filter," Optics Letters 18:1370-1372 (1993)

[6] T. A. Berkoff and A. D. Kersey, "Fiber Bragg grating array sensor system using a bandpass wavelength division multiplexer and interferometric detection," Phot. Tech. Lett. 8(11):1522-1524 (1996)

[7] G. A. Johnson, M. D. Todd, B. L. Aulthouse and C. C. Chang, "Fiber Bragg grating interrogation and multiplexing with a $3 \times 3$ coupler and a scanning filter," J. Lightwave Tech. 18(8):1101-1105 (2000)

[8] M. D. Todd, G. A. Johnson and C. C. Chang, "Passive, light intensity-independent interferometric method for fibre Bragg grating interrogation," Electronics Letters 35(22):1970-1971 (1999)

[9] R. S. Weis, A. D. Kersey and T. A. Berkoff, "A four-element fiber grating sensor array with phase-sensitive detection," J. Lightwave Tech. 6(12):1469-1472 (1994)

[10] P. Orr and P. Niewczas, "High-speed, solid state, interferometric interrogator and multiplexer for fiber Bragg grating sensors," J. Lightwave Tech. 29(22):3387-3392 (2011)

[11] B. Lee, "Review of the present status of optical fiber sensors," Optical Fiber Technology 9:57-79 (2003)

[12] F. Schliep, R. Hereth and G. Schiffner, "Phase sensitive investigations of $3 \times 3$ singlemode fibre directional couplers," Electronics Letters 29(1):68-70 (1993)

[13] W.C. Swann, M.A. Hubbard, and S.L. Gilbert, "Hybrid multiple wavelength reference using fiber gratings and molecular absorption," in Proc. Bragg Gratings, Photosensitivity, and Poling in Glass Waveguides, OSA Technical Digest, pp. 63-65 (1999) 